Address correspondence to: Gregory M. Vercellotti, University of Minnesota, 420 Delaware St. SE, MMC 480, Minneapolis, Minnesota 55455, USA. Phone: 612.626.3757; Fax: 612.625.6919; E-mail: verce001@umn.edu.

1. Herrick JB. Peculiar elongated and sickle-shaped red blood corpuscles in a case of severe anemia. Arch Intern Med. 2010;6(5):517-521.

2. Hebbel RP, Yamada O, Moldow CF, Jacob HS, White JG, Eaton JW. Abnormal adherence of sickle erythrocytes to cultured vascular endothelium: possible mechanism for microvascular occlusion in sickle cell disease. J Clin Invest. 1980;65(1):154-160.

3. Turhan A, Weiss LA, Mohandas N, Coller BS, Frenette PS. Primary role for adherent leukocytes in sickle cell vascular occlusion: a new paradigm. Proc Natl Acad Sci U S A. 2002;99(5):3047-3051.

4. Hidalgo A, Chang J, Jang JE, Peired AJ, Chiang EY, Frenette PS. Heterotypic interactions enabled by polarized neutrophil microdomains mediate thromboinflammatory injury. Nat Med. 2009;15(4):384-391.

5. Belcher JD, Marker PH, Weber JP, Hebbel RP, Vercellotti GM. Activated monocytes in sickle cell disease: potential role in the activation of vascular endothelium and vaso-occlusion. Blood. 2000;96(7):2451-2459

6. Brittain JE, Knoll CM, Ataga KI, Orringer EP, Parise LV. Fibronectin bridges monocytes and reticulocytes via integrin $\alpha 4 \beta 1$. Br J Haematol. 2008;141(6):872-881.

7. Brittain HA, Eckman JR, Swerlick RA, Howard RJ,
Wick TM. Thrombospondin from activated platelets promotes sickle erythrocyte adherence to human microvascular endothelium under physiologic flow: a potential role for platelet activation in sickle cell vaso-occlusion. Blood. 1993;81(8):2137-2143.

8. Wallace KL, Linden J. Adenosine A2A receptors induced on iNKT and NK cells reduce pulmonary inflammation and injury in mice with sickle cell disease. Blood. 2010;116(23):5010-5020.

9. Kaul DK, Hebbel RP. Hypoxia/reoxygenation causes inflammatory response in transgenic sickle mice but not in normal mice. J Clin Invest. 2000;106(3):411-420.

10. Zennadi R, et al. Erythrocyte plasma membranebound ERK1/2 activation promotes ICAM-4-mediated sickle red cell adhesion to endothelium. Blood. 2012;119(5):1217-1227.

11. Belcher JD, et al. Heme triggers TLR4 signaling leading to endothelial cell activation and vasoocclusion in murine sickle cell disease. Blood. 2014;123(3):377-390

12. Fabry ME, et al. High expression of human beta S- and alpha-globins in transgenic mice: erythrocyte abnormalities, organ damage, and the effect of hypoxia. Proc Natl Acad Sci U S A. 1992;89(24):12155-12159.

13. Paszty C, et al. Transgenic knockout mice with exclusively human sickle hemoglobin and sickle cell disease. Science. 1997;278(5339):876-878.

14. Wu LC, Sun CW, Ryan TM, Pawlik KM, Ren J, Townes TM. Correction of sickle cell disease by homologous recombination in embryonic stem cells. Blood. 2006;108(4):1183-1188.

15. Kato GJ, Hebbel RP, Steinberg MH, Gladwin MT. Vasculopathy in sickle cell disease: biology, pathophysiology, genetics, translational medi- cine, and new research directions. Am J Hematol. 2009;84(9):618-625.

16. Fuchs TA, Wagner DD, Frenette PS. Heme-induced neutrophil extracellular traps (NETs) formation contributes to sickle cell disease pathogenesis. Blood. 2013;122(21):184.

17. Mathson K, Teena R, Belcher JD, Vercellotti GM, Slungaard A. Thiocyanate blocks peroxidasedependent extracellular trap (ET) formation by PMN and eosinophils: heme is a potent new agonist for the ET pathway. Blood. 2013;122(21):323.

18. Jakubowski JA, et al. A phase 1 study of prasugrel in patients with sickle cell disease: effects on biomarkers of platelet activation and coagulation. Thromb Res. 2014;133(2):190-195.

19. Desai PC, et al. A pilot study of eptifibatide for treatment of acute pain episodes in sickle cell disease. Thromb Res. 2013;132(3):341-345.

20. Chang J, Patton JT, Sarkar A, Ernst B, Magnani JL, Frenette PS. GMI-1070, a novel pan-selectin antagonist, reverses acute vascular occlusions in sickle cell mice. Blood. 2010;116(10):1779-1786.

21. Li J, et al. Neutrophil AKT2 regulates heterotypic cell-cell interactions during vascular inflammation. J Clin Invest. 2014;124(4):1483-1496.

22. Cho $\mathrm{H}$, et al. Insulin resistance and a diabetes mellitus-like syndrome in mice lacking the protein kinase Akt2 (PKB $\beta$ ). Science. 2001;292(5522):1728-1731.

23 . Belcher JD, et al. Heme oxygenase-1 gene delivery by Sleeping Beauty inhibits vascular stasis in a murine model of sickle cell disease. J Mol Med (Berl). 2010;88(7):665-675

24. Kalambur VS, et al. Microvascular blood flow and stasis in transgenic sickle mice: utility of a dorsal skin fold chamber for intravital microscopy. Am J Hematol. 2004;77(2):117-125.

\title{
Insulin, osteoblasts, and energy metabolism: why bone counts calories
}

\author{
Ryan C. Riddle and Thomas L. Clemens \\ Department of Orthopaedic Surgery, Johns Hopkins University School of Medicine, Baltimore, Maryland, USA. \\ Baltimore Veterans Administration Medical Center, Baltimore, Maryland, USA.
}

\begin{abstract}
Recent studies have demonstrated that insulin stimulates bone cells to produce and activate osteocalcin, an endocrine hormone that increases the efficiency of glucose metabolism through its actions on the pancreas and other peripheral tissues. In this issue of the JCI, Wei and colleagues directly explore the contribution of insulin signaling in osteoblasts to the disturbances in whole-body glucose metabolism associated with a high-fat diet. In mice fed a high-fat diet, increased uptake of saturated fatty acids by the osteoblast accelerates the ubiquitination and degradation of the insulin receptor. In this setting, impairments in osteoblast insulin signaling reduce serum levels of undercarboxylated osteocalcin, which in turn exacerbate insulin resistance in muscle and white adipose tissue. These findings underscore the importance of insulin-responsive skeletal cells as components of a newly appreciated endocrine network critical for regulating global energy homeostasis.
\end{abstract}

Conflict of interest: The authors have declared that no conflict of interest exists.

Citation for this article: J Clin Invest. 2014; 124(4):1465-1467. doi:10.1172/JCI75554.

\section{Bone as a metabolic organ: lessons from evolution}

The evolution of a large appendicular skeleton powered by robust skeletal muscles in early tetrapods was a successful strategy for ambulation on land. Additionally, the new skeleton served as a repository for calcium, a scarce commodity in the terrestrial habitat, and the emergence of the parathyroid gland at this juncture provided the means to rapidly access bone calcium through osteoclast-mediated liberation from skeletal stores (1). However, the upgraded musculoskeletal system also increased overall fuel requirements and altered global energy balance, prompting the evolution of other endocrine networks to coordinate energy expenditure (2). Prominent among these emerging networks were the leptin and insulin/insulin-like growth factor pathways, which have assumed central roles in growth and metabolism in higher organisms $(3,4)$. The importance of these meta- 
bolic pathways in modern mammals is illustrated by common metabolic diseases, such as osteoporosis, diabetes, and obesity, caused by genetic or environmental disturbances in endocrine control mechanisms.

In contrast to the large number of studies on energy metabolism in muscle and adipose tissue, surprisingly little attention has been paid to understanding the bioenergetics of bone metabolism. The sheer size of the skeleton alone implies that its energy requirements should have an impact on global metabolic demands, particularly during growth and remodeling. Indeed, mature osteoblasts that are actively synthesizing and mineralizing matrix exhibit abundant mitochondria, consistent with the increased metabolic demand during this active phase of their life span (5). Likewise, osteocytes, which represent terminally differentiated osteoblasts, survive for years embedded in mineralized bone at densities of greater than 10,000 cells per cubic millimeter. These cells are the main source of the phosphate-regulating hormone FGF23 (6) and maintain an extensive lacunar-canalicular network that interconnects bone cells throughout the skeleton.

The first clues that bone might participate in metabolic homeostasis came from studies by Ducy and colleagues, who demonstrated that leptin alters bone mass through a hypothalamic relay (7). Further work led to the recognition that leptin's central effect on the osteoblast also contributes to the hormone's influence on insulin secretion (8). More recently, insulin signaling in the osteoblast was found to be required for proper glycemic control in mice $(9,10)$. Indeed, disturbances in glucose homeostasis, including glucose intolerance and insulin resistance, in mice specifically lacking Insr in osteoblasts could be largely corrected by treatment with bioactive, undercarboxylated osteocalcin (ucOCN); therefore, insulin receptor signaling in osteoblasts increases the production and bioavailability of osteocalcin, which is made exclusively by osteoblasts and osteocytes, and acts in an endocrine fashion to regulate pancreatic insulin secretion and peripheral insulin responsiveness $(11,12)$. These observations strongly suggest that osteoblasts and osteocytes participate in the normal control of global glucose homeostasis.

A model of insulin resistance in bone In the current issue of the JCI, Wei and colleagues (13) use both genetic and physiologic approaches to more directly probe the characteristics of the osteoblast insulin receptor in the context of diet-induced disturbances in metabolism. Mice engineered to modestly overexpress (via the Col1a1 promoter) or underexpress (via osteoblastspecific Insr haploinsufficiency) the insulin receptor in osteoblasts were metabolically normal until challenged with a high-fat diet (HFD). Under these conditions, mice overexpressing the insulin receptor in osteoblasts exhibited better glucose tolerance and were more responsive to insulin than controls, whereas Insr-underexpressing mice exhibited more severe glucose intolerance compared with that of control animals. In accordance with previous studies linking insulin-dependent effects on osteocalcin bioavailability to osteoclastmediated decarboxylation and release from the bone matrix (10), bone resorption and serum ucOCN were reduced in WT mice fed a HFD, while mice with increased or decreased insulin signaling in osteoblasts had greater or lesser amounts of ucOCN, respectively. Importantly, Wei et al. revealed that reduced insulin sensitivity of bone also contributes to the glucose intolerance seen in normal mice fed a HFD.

Remarkably, Wei and colleagues (13) found that osteoblasts from HFD-fed mice exhibit features of insulin resistance that closely resemble those seen in the liver and muscle. These include a reduced ability of insulin to stimulate phosphorylation of IRS1/2, a defect that has been linked to increases in circulating and tissue levels of saturated, lipotoxic fatty acids that raise intracellular diacylglycerol levels (14). To explore the link between lipotoxicity and dysfunctional insulin signaling in bone, Wei et al. treated primary osteoblasts with saturated fatty acids and confirmed an inhibitory effect on insulin receptor signaling. Intriguingly, stearate in particular caused a reduction in insulin receptor abundance due to increased SMURF-dependent ubiquitination, suggesting a unique pathway for the development of insulin resistance in bone.

\section{Implications and perspectives}

The studies by Wei et al. (13) strengthen the case for a link between bone and energy glucose metabolism, in which the insulin receptor and osteocalcin are central components. Wei and colleagues favor a mechanism involving insulin's ability to increase the bioavailability of the undercarboxylated form of osteocalcin, which in turn facilitates uptake and metabolism of glucose by adipose and muscle; however, the mechanism whereby ucOCN facilitates glucose uptake by muscle and adipose is unclear. A putative receptor for ucOCN, GPCR6A, has been identified and implicated as the mediator of ucOCN action on the pancreas and testicular Leydig cells (15), though it remains to be determined whether this same receptor mediates other insulin-sensitizing actions. Indeed, recent examination of central actions of osteocalcin suggests the existence of another receptor, which apparently mediates certain behavioral actions of osteocalcin (16).

As acknowledged by Wei et al., it seems highly likely that insulin signaling in osteoblasts also affects global glucose homeostasis though osteocalcin-independent mechanisms. For example, insulin is known to stimulate glucose uptake by osteoblasts (17), which express several high-affinity insulin-responsive glucose transporters (18); therefore, any loss of insulin-dependent glucose uptake by osteoblasts (e.g., high-fat intake) could have a profound affect on whole-body glucose disposal and contribute to the disturbances in glucose homeostasis. Studies to precisely define the relative contribution of bone cells to whole-body glucose disposal will require more rigorous approaches, such as the use of euglycemic clamps. Finally, very recent studies show that the ability of WNT/ LRP5 signaling to promote osteoblast differentiation depends in part on the ability of osteoblasts to activate key glycolytic enzymes that promote aerobic glycolysis (19). Such data indicate that factors other than insulin play a role in the regulation glucose utilization by osteoblasts.

In summary, the studies by Wei et al. (13) add to a growing body of data that implicate bone and its cells as an important metabolic organ that is functionally linked to other metabolically active tissues by common endocrine hormones, such as insulin. Future studies are needed to more precisely define the fraction of the whole-body caloric intake that is required for bone cells to function relative to that required by other tissues. In addition, it would seem important to know what types of fuels are used by osteoblasts and whether fuel preferences vary according to different functional demands of osteoblasts at different stages of their life cycle. From a clinical perspective, studies investigating the possibility that metabolic disturbances underlying the pathogenesis of diabetes and obesity might also affect the skeleton and vice versa are 
already underway. Answers to such questions will certainly expand our understanding of the biology of the skeleton and might ultimately aid in the diagnosis and management of patients with a broad range of metabolic diseases.

\section{Acknowledgments}

R.C. Riddle is supported by a grant from the National Institute of Diabetes and Digestive and Kidney Diseases of the NIH (DK099134) and a Career Development Award from the Veterans Administration. T.L. Clemens is supported by a Merit Review grant (BX001234) and is the recipient of a Research Career Scientist Award from the Veterans Administration.

Address correspondence to: Thomas L. Clemens, Department of Orthopaedic Surgery, Johns Hopkins University School of Medicine, 601 N. Caroline St., Baltimore, Maryland 21287, USA. Phone: 410.955.3245; Fax: 410.614.1451; E-mail: tclemen5@jhmi.edu.
1. Okabe M, Graham A. The origin of the parathyroid gland. Proc Natl Acad Sci U S A. 2004; 101(51):17716-17719.

2. DiGirolamo DJ, Clemens TL, Kousteni S. The skeleton as an endocrine organ. Nat Rev Rheumatol. 2012;8(11):674-683.

3. Tatar M, Bartke A, Antebi A. The endocrine regulation of aging by insulin-like signals. Science. 2003;299(5611):1346-1351.

4. Denver RJ, Bonett RM, Boorse GC. Evolution of leptin structure and function. Neuroendocrinology. 2011;94(1):21-38.

5. Pritchard JJ. A cytological and histochemical study of bone and cartilage formation in the rat. J Anat. 1952;86(3):259-277.

6. Martin A, David V, Quarles LD. Regulation and function of the FGF23/klotho endocrine pathways. Physiol Rev. 2012;92(1):131-155.

7. Ducy $\mathrm{P}$, et al. Leptin inhibits bone formation through a hypothalamic relay: a central control of bone mass. Cell. 2000;100(2):197-207.

8. Hinoi E, et al. The sympathetic tone mediates leptin's inhibition of insulin secretion by modulating osteocalcin bioactivity. J Cell Biol. 2008;183(7):1235-1242.

9. Fulzele K, et al. Insulin receptor signaling in osteoblasts regulates postnatal bone acquisition and body composition. Cell. 2010;142(2):309-319.

10. Ferron $\mathrm{M}$, et al. Insulin signaling in osteoblasts integrates bone remodeling and energy metabolism. Cell. 2010;142(2):296-308.

11 . Lee NK, et al. Endocrine regulation of energy metab- olism by the skeleton. Cell. 2007;130(3):456-469.

12. Ferron M, McKee MD, Levine RL, Ducy P, Karsenty G. Intermittent injections of osteocalcin improve glucose metabolism and prevent type 2 diabetes in mice. Bone. 2011;50(2):568-575.

13. Wei J, et al. Bone-specific insulin resistance disrupts whole-body glucose homeostasis via decreased osteocalcin activation. J Clin Invest. 2014; 124(4):1781-1793.

14. Farese RV, et al. Muscle-specific knockout of PKClambda impairs glucose transport and induces metabolic and diabetic syndromes. J Clin Invest. 2007;117(8):2289-2301.

15. Karsenty G. Bone endocrine regulation of energy metabolism and male reproduction. C R Biol. 2011;334(10):720-724.

16. Oury F, et al. Maternal and offspring pools of osteocalcin influence brain development and functions. Cell. 2013;155(1):228-241.

17. Hahn TJ, Westbrook SL, Sullivan TL, Goodman WG, Halstead LR. Glucose transport in osteoblastenriched bone explants: characterization and insulin regulation. J Bone Miner Res. 1988;3(3):359-365.

18. Zoidis E, Ghirlanda-Keller C, Schmid C. Stimulation of glucose transport in osteoblastic cells by parathyroid hormone and insulin-like growth factor I. Mol Cell Biochem. 2011;348(1-2):33-42.

19. Esen E, Chen J, Karner CM, Okunade AL, Patterson BW, Long F. WNT-LRP5 signaling induces Warburg effect through mTORC2 activation during osteoblast differentiation. Cell Metab. 2013;17(5):745-755. 